\title{
Turing Patterns and Waves in Closed Two-Layer Gel Reactors
}

\author{
Brigitta Dúzs, ${ }^{\dagger}$ Patrick De Kepper, ${ }^{\ddagger}$ and István Szalai*, ${ }^{\dagger}$ \\ ${ }^{\dagger}$ Institute of Chemistry, Eötvös Loránd University, Pázmány Péter s. 1/A, H-1117 Budapest, Hungary \\ ${ }^{\ddagger}$ Centre de Recherche Paul Pascal, CNRS, University of Bordeaux I, Avenue Schweitzer, F-33600 Pessac, France
}

Supporting Information

ABSTRACT: Reaction-diffusion waves and stationary Turing patterns are observed in closed two-layer gel reactors, where the two compartments are initially filled with complementary sets of reactants of the chlorine dioxideiodine-malonic acid-poly(vinyl alcohol) reaction. The asymmetrical loading generates concentration gradients and the patterns form at the interface between the two parts. These easy-to-perform experiments allow us to study a wide range of dynamical phenomena without requiring a specific reactor design or the use of sophisticated equipment. To get complementary information on pattern formation in parallel and perpendicular to the direction of the concentration gradients, two geometrically different configurations of compartments are presented. We demonstrate that three variants of the initial distribution of the chemicals can be equally applied, and this flexibility provides a way to introduce additional reagents to perturb the dynamics of the systems. A noticeable increase in the wavelength of Turing patterns and in the period of waves has been induced by adding bromide ions. The interaction of Turing and Hopf modes has been observed as a result of not only the variation of the initial poly(vinyl alcohol) concentration but that of the gradients as well.
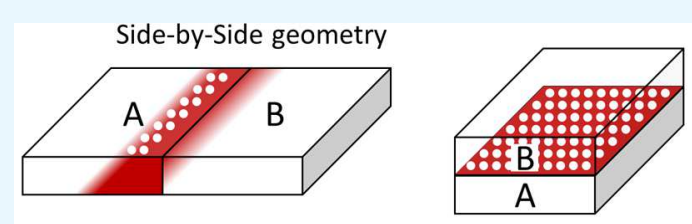

Top

and

Bottom

geometry
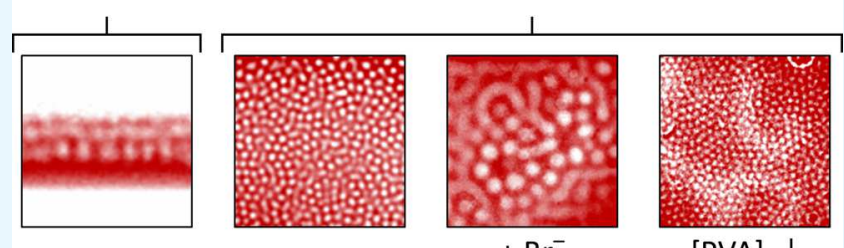

$[\mathrm{PVA}]_{0} \downarrow$

\section{INTRODUCTION}

The conscious design of complex chemical systems, which can be used to model and mimic natural biology, is a challenging problem. ${ }^{1-3}$ Nonlinear reactions and diffusive transport are often used as building blocks of such development. The thoughtful study of reaction-diffusion (RD) systems requires the use of well-defined reactor configurations, which allow us to investigate different aspects of the pattern formation dynamics, for example, the sensitivity to the initial and boundary conditions. The chlorite-iodide-malonic acid (CIMA) and its variant, the chlorine dioxide-iodine-malonic acid (CDIMA) batch chemical oscillators, ${ }^{4}$ are preferably used to investigate $\mathrm{RD}$ phenomena because of their well-described kinetics and advantageous experimental characteristics such as robustness and reproducibility. The CIMA and CDIMA RD systems have been typically studied in open spatial gel reactors which allow us to maintain the system at controlled distance from thermodynamic equilibrium. ${ }^{5}$ Although, these experiments require sophisticated equipment and special constructions, the formation of sustained structures facilitates the evaluation of the results. The most often used open reactor configurations are the one-side-fed reactor (OSFR) and twoside-fed reactor (TSFR), where the RD medium, for example, a piece of hydrogel, is fed by fresh chemicals from one or two outer compartments. In an OSFR, the gel exchanges chemicals through a single face with an open reservoir, which is fed by the initial reagents. In a TSFR, the gel is sandwiched between two open tanks, which are supplied by complementary sets of reactants. TSFRs have been used to create patterns in the CIMA RD system, ${ }^{6-10}$ whereas in the CDIMA case, the OSFR geometry has been preferred. ${ }^{11-13}$ These two geometries have different advantages and disadvantages, ${ }^{5}$ but versatile $\mathrm{RD}$ patterns, for example, 2D and 3D spot patterns, symmetric triangles, hexabands, stripes, antisynchronous waves, spirals, and Turing-Hopf spirals, have been found in both of them.

Experiments in closed reactors definitely require simpler setups but allow only the formation of transient phenomena. Because of the special batch oscillatory properties of the CDIMA and CIMA reactions, trigger waves, ${ }^{14}$ interacting hexagons, and network-like structures ${ }^{15}$ can be observed in a thin, unstirred liquid layer, for example, in a Petri dish. Recently, a new configuration where two pieces of gel are loaded homogeneously with subsets of a nonlinear chemical system has been suggested to study the formation of a localized pattern zone in a gradient of chemical composition. ${ }^{16,17}$ The reactants meet by diffusion and a reaction zone develops at the cross-gradients of the chemicals. A closed two-layer setup can be easily mounted which facilitates its widespread use, and the complementary gel halves serve as transitory reagent sources for the middle reaction zone. This provides better control on the dynamics of the patterning zone, contrary to an unstirred liquid layer, and enables to maintain the self-organized

Received: October 29, 2018

Accepted: January 10, 2019

Published: February 14, 2019 
structures for considerably longer times. In this configuration, the dynamics of autocatalytic and oscillatory-type reacting systems have been investigated. ${ }^{18}$ According to the theoretical and the corresponding numerical studies, the formation of fronts, spatially localized waves, Turing patterns, and complex spatiotemporal behavior is predicted as a result of the interaction of the Hopf and Turing modes. ${ }^{16,17}$ The front and wave dynamics could be realized in appropriate experiments by using the Belousov-Zhabotinsky (BZ) reaction, ${ }^{18}$ but was not possible for Turing-type patterns with this reaction. Here, we investigate the CDIMA reaction which appeared as a good candidate to produce not only waves but also Turing patterns in such closed two-layer setups.

The core of the mechanism of the CDIMA reaction can be written by reactions (R1-R3). The model ${ }^{19}$ describes a substrate inhibition mechanism, where iodide and chlorite play the role of the activator and the inhibitor, respectively.

$$
\mathrm{MA}+\mathrm{I}_{2} \rightarrow \mathrm{IMA}+\mathrm{I}^{-}+\mathrm{H}^{+}
$$

Here MA and IMA for for malonic acid and iodomalonic acid.

$$
\begin{aligned}
& \mathrm{ClO}_{2}+\mathrm{I}^{-} \rightarrow \mathrm{ClO}_{2}^{-}+0.5 \mathrm{I}_{2} \\
& \mathrm{ClO}_{2}{ }^{-}+4 \mathrm{I}^{-}+4 \mathrm{H}^{+} \rightarrow \mathrm{Cl}^{-}+2 \mathrm{I}_{2}+2 \mathrm{H}_{2} \mathrm{O} \\
& \mathrm{I}_{2}+\mathrm{I}^{-} \rightleftharpoons \mathrm{I}_{3}^{-}
\end{aligned}
$$

This system is capable of forming stationary Turing patterns in $\mathrm{RD}$ systems if the effective relative diffusivity of the $\mathrm{I}^{-}$species is decreased by immobilizing macromolecular agents such as starch or poly(vinyl alcohol) (PVA), which are capable of complexing $\mathrm{I}_{3}{ }^{-}$ions formed in $\mathrm{R} 4$.

In this work, $\mathrm{I}_{2}, \mathrm{MA}$, and in some cases $\mathrm{ClO}_{2}$ are the initially separated components, whereas the other reactants $\left(\mathrm{H}_{2} \mathrm{SO}_{4}\right.$ and PVA) are distributed equally in both sides. We study three types of initial conditions depending on the placing of $\mathrm{ClO}_{2}$, as it can be introduced equally on both sides (Figure 1a) or to either of the sides (Figure 1b,c). The two sides are homogeneously loaded with the indicated reactants, and when these are connected, counter-diffusion leads to the formation of a reaction zone in the middle. In this zone, the initial separation of $I_{2}$ and MA evokes the localized formation of the activator in reaction $\mathrm{R} 1$, which produces a reversible complex complex with PVA. Because the effective diffusivity of $\mathrm{ClO}_{2}$ remains unchanged, it fills up the middle zone and exerts inhibition ( $\mathrm{R} 2$ and $\mathrm{R} 3$ ) both in symmetric and in asymmetric initial $\mathrm{ClO}_{2}$ distributions. However, the elimination of $\mathrm{ClO}_{2}$ from one side enables to introduce other species (e.g., halide ions ${ }^{20}$ ) to that side to affect the dynamics. The patterns of the middle zone have been studied from two different directions, perpendicular and parallel to the concentration gradients in the side-by-side ( $\mathrm{SbS}$, Figure 1e) and in the top-and-bottom $(\mathrm{TaB}$, Figure 1f) configurations. We present an experimental route to generate the theoretically predicted ${ }^{16}$ waves and stationary Turing-type patterns in the closed two-layer configurations by using the CDIMA reaction, a reaction that can produce batch oscillatory dynamics as the $\mathrm{BZ}$ reaction.

\section{RESULTS AND DISCUSSION}

We start with the effects of the initial distribution of the chemicals between the two gels, especially the placing of $\mathrm{ClO}_{2}$. Prior to any reactions, without $\mathrm{ClO}_{2}$, side $\mathrm{A}$ is reddish-purple $\left(\lambda_{\max }=490 \mathrm{~nm}\right.$, dark in the pictures, Figure $2 \mathrm{~b}$ ) because of the PVA $-\mathrm{I}_{3}{ }^{-}$complex, which is formed in a small amount thanks (a)

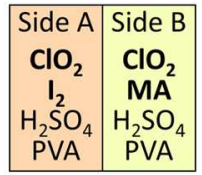

(b)

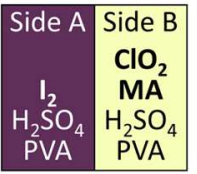

(c)

\begin{tabular}{|c|c|}
\hline Side A & Side B \\
$\mathrm{ClO}_{2}$ & \\
$\mathrm{I}_{2}$ & $\mathrm{MA}$ \\
$\mathrm{H}_{2} \mathrm{SO}_{4}$ & $\mathrm{H}_{2} \mathrm{SO}_{4}$ \\
$\mathrm{PVA}$ & $\mathrm{PVA}^{-}$ \\
\hline
\end{tabular}

(e)

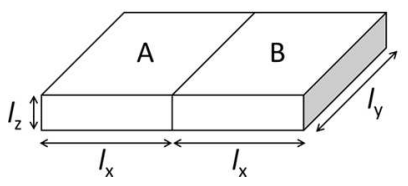

(d)
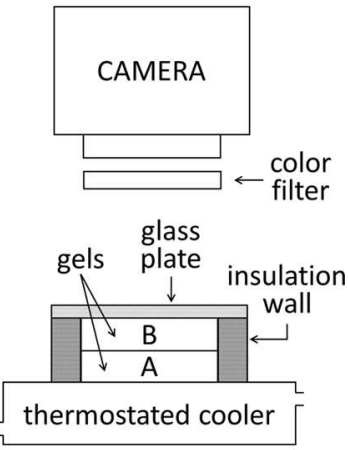

\begin{tabular}{|c|}
$\mid$ |। | | | | | | | | | | | | \\
\hline LED light source \\
\hline
\end{tabular}

(f)

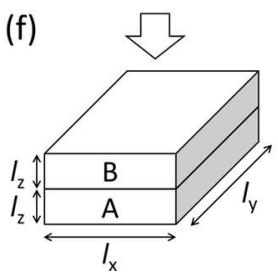

Figure 1. Sketch of the closed two-layer gel reactors used in the experiments. The initial reagent distributions $(\mathrm{a}-\mathrm{c})$, the realization of the setup (d), and the experimental configurations: SbS (e) and TaB (f) with the direction of observation (indicated by arrows). Sides A and $\mathrm{B}$ are homogeneously loaded with different sets of reactants of the CDIMA reaction and connected to start the experiment. The dimensions of the gel pieces are: $l_{x}=16 \mathrm{~mm}, l_{y}=30 \mathrm{~mm}$, and $l_{z}=$ $2.8 \mathrm{~mm}$. (a)

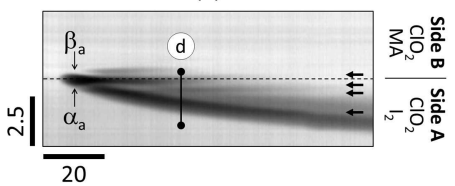

(b)

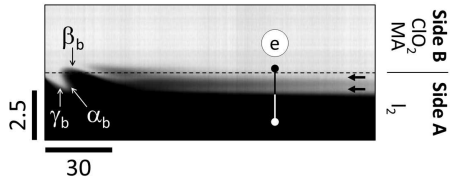

(c)

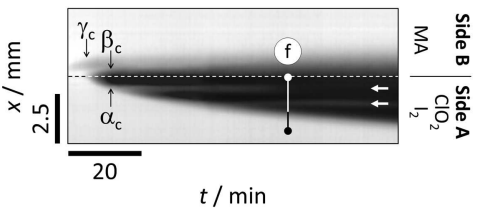

(d)

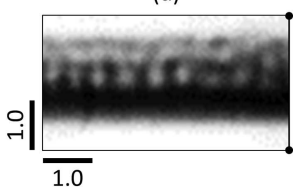

(e)

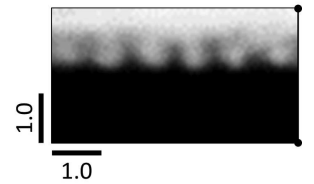

(f)

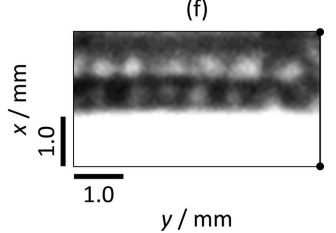

Figure 2. Results in the $\mathrm{SbS}$ experimental setup. Time-space plots (from $t=0$ ) in the case of different initial $\mathrm{ClO}_{2}$ distributions $(\mathrm{a}-\mathrm{c})$, where the arrows indicate the rows of stationary structures. The dotended lines show the $(x, t)$ sections of the corresponding figures of $(d-f)$. Snapshots of the forming multilayer spot-like patterns in the $x-y$ plane at $t=45 \mathrm{~min}(\mathrm{~d}), t=103 \mathrm{~min}(\mathrm{e})$, and $t=60 \mathrm{~min}(\mathrm{f})$. Concentrations: $[\mathrm{PVA}]_{0}=3 \mathrm{~g} / \mathrm{L}$.

to the $\mathrm{I}^{-}$traces in the iodine solution. If $\mathrm{ClO}_{2}$ is added to side A, it oxidizes nearly all the $\mathrm{I}^{-}$, and the intense color of the 
(a)

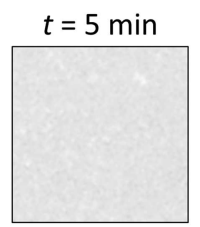

(b)

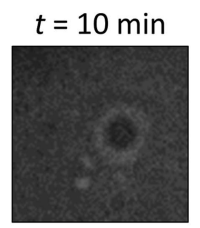

(c)

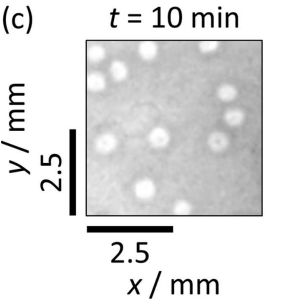

$15 \mathrm{~min}$

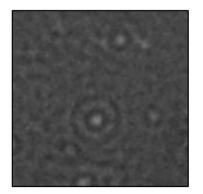

$15 \mathrm{~min}$

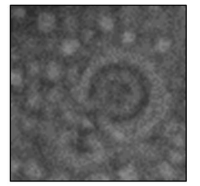

$20 \mathrm{~min}$

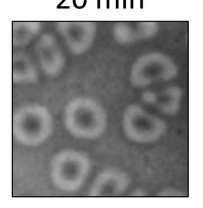

$20 \mathrm{~min}$

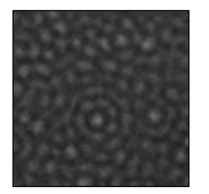

$20 \mathrm{~min}$

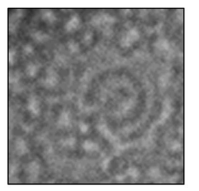

$30 \mathrm{~min}$

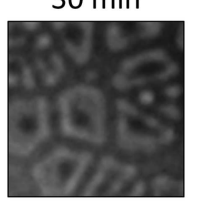

$30 \mathrm{~min}$
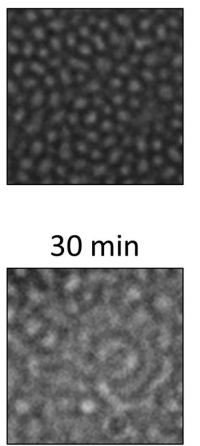

$40 \mathrm{~min}$

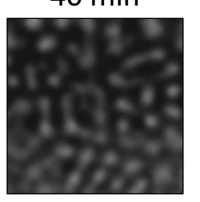

$50 \mathrm{~min}$
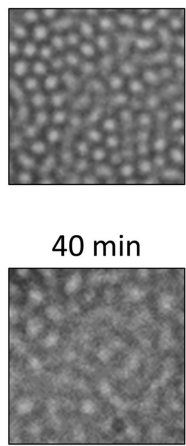

$50 \mathrm{~min}$

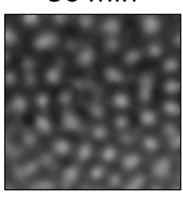

$60 \mathrm{~min}$

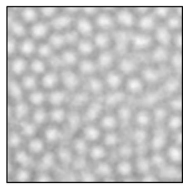

$50 \mathrm{~min}$

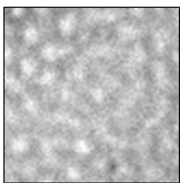

$60 \mathrm{~min}$

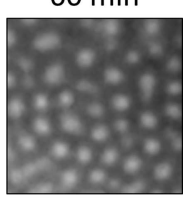

Figure 3. Results in the $\mathrm{TaB}$ experimental setup: The formation and stabilization of hexagonally ordered spots is presented on consecutive snapshots in the cases when $\mathrm{ClO}_{2}$ is on both sides (a), when $\mathrm{ClO}_{2}$ is added only to side $\mathrm{B}$ (b), and when $\mathrm{ClO}$ is added only to side $\mathrm{A}$ (c). Concentrations: $[\mathrm{PVA}]_{0}=3 \mathrm{~g} / \mathrm{L}$.

complex disappears. In this case, the color of side $\mathrm{A}$ is orangish (light in the pictures, Figure 2a,c). Side B is slightly greenishyellow because of $\mathrm{ClO}_{2}$, which appears to be of the same light color in the pictures (Figure $2 a-c$ ). On the time scale of the filling of the gel pieces (about $3 \mathrm{~h}$ ), we have not observed any other significant reactions in side A nor $\mathrm{B}$.

The experiment starts when side $\mathrm{A}$ and $\mathrm{B}$ are connected $(t=$ $0)$. At first, we discuss the results found in the SbS configuration (Figure 1e). In the case, when $\mathrm{ClO}_{2}$ is present in both sides, the $\mathrm{I}^{-}$production leads to the formation of a dark reaction zone in the middle, bounded by a pair of fronts indicated by $\alpha$ and $\beta$ in Figure 2a. The $\beta$-type front, which is less sharp and propagates into side $\mathrm{B}$, can be identified in all the used distributions of $\mathrm{ClO}_{2}$ (Figure $2 \mathrm{a}-\mathrm{c}$ ). On the contrary, front $\alpha$ cannot be seen in the case when $\mathrm{ClO}_{2}$ is added only to side $B$ (Figure $2 \mathrm{~b}$ ). In every case, because of the applied $[\mathrm{MA}]_{0} /\left[\mathrm{I}_{2}\right]_{0}$ ratio, the visible reaction zone propagates to the direction of side $\mathrm{A}$. However, when the $\mathrm{ClO}_{2}$ distribution is asymmetric, a prior front $\left(\gamma_{\mathrm{b}}\right.$ or $\gamma_{\mathrm{c}}$ in Figure $\left.2 \mathrm{~b}, \mathrm{c}\right)$ develops following the direction of the diffusion of $\mathrm{ClO}_{2}$. The preliminary front starts immediately at the beginning of the experiment and is followed by $\alpha$ and $\beta$ with a time-lag of 5-7 min, which is typical in all cases. According to the observed color, fronts $\alpha, \beta$, and $\gamma_{\mathrm{c}}$ are characterized by $\mathrm{I}^{-}$production. On the contrary, $\gamma_{\mathrm{b}}$ is governed by the diffusion of $\mathrm{ClO}_{2}$ toward side $\mathrm{A}$, and the light color is the result of the consumption of the $\mathrm{I}^{-}$traces by $\mathrm{ClO}_{2}$ (Figure 2c). The concentration gradients determine the spatial extent of the range of Turing instability in all the three cases. This domain gets wider in time, and the stationary structures emerge in succeeding rows along axis $x$ as it is shown by the parallel light stripes indicated by arrows in Figure $2 \mathrm{a}-\mathrm{c}$. The Turing structures disappear after about $80-120 \mathrm{~min}$ at the applied conditions. The stabilized multilayer spot-like structures in the $x-y$ plane are shown in Figure $2 \mathrm{~d}-\mathrm{f}$. In the case of symmetric $\mathrm{ClO}_{2}$ distribution, the wavelength of the forming pattern is smaller, typically $0.54 \pm 0.07 \mathrm{~mm}$ (Figure $2 \mathrm{~d}$ ) compared to the other cases, where it is about $0.81 \pm 0.06$ and $0.71 \pm 0.07 \mathrm{~mm}$ (Figure 2e,f), so the zone of Turing instability consists of more rows of spots at different positions along axis $x$ than in the latter two cases. This is in accordance with the prior results made in open spatial gel reactors, where the wavelength of the pattern increased as the input feed concentration of $\mathrm{ClO}_{2}$ was decreased. $^{11}$ In the closed two-layer setup, the initial asymmetric $\mathrm{ClO}_{2}$ distribution results in smaller local $\mathrm{ClO}_{2}$ concentration in the reaction zone compared to the symmetric one.

The patterns forming in the plane perpendicular to the direction of concentration gradients have been analyzed in the $\mathrm{TaB}$ geometry (Figure 1f). In this geometry, the recorded pictures are the superposition of the three-dimensional patterns which form in different layers of the gel. ${ }^{21}$ In all the three cases of the initial $\mathrm{ClO}_{2}$ distribution, we have found experimental evidence of spotlike hexagonal patterns (Figure 3). However, in Figure 3b, the patterns cannot be stabilized and are less visible because of the high color contrast between sides $\mathrm{A}$ and $\mathrm{B}$. The spots can evolve from concentric target-like patterns (Figure $3 \mathrm{a}, t=15 \mathrm{~min}$ ) or from a cellular structure (Figure $3 \mathrm{c}, t=30 \mathrm{~min}$ ), but the time needed to reach a quasiregularly ordered pattern is almost the same $(50 \mathrm{~min})$ in both cases. The wavelength of the spotlike structures typically does not change during the stabilization as can be seen, for example, by comparing the snapshots taken at 30 and $60 \mathrm{~min}$ in Figure 3a. In accordance with the SbS measurements, in cases of asymmetric $\mathrm{ClO}_{2}$ distributions, the wavelength of the forming pattern is about 1.5 times larger than in the case of the symmetric $\mathrm{ClO}_{2}$ distribution [compare $\lambda=0.73 \pm 0.07 \mathrm{~mm}$ at $t=60 \mathrm{~min}$ (Figure $3 \mathrm{c}$ ) with $\lambda=0.54 \pm 0.08 \mathrm{~mm}$ at $t=50 \mathrm{~min}$ (Figure 3a)].

At this point, we are ready to test the recently published method of adding halide ions to the CDIMA system to increase the wavelength of the Turing patterns. ${ }^{20}$ Although, it was originally used to modify patterns in a one-side-fed open reactor, we found that it can be applied in closed two-layer 
systems as well. We have chosen $\mathrm{Br}^{-}$ions, which were added to the $\mathrm{ClO}_{2}$-free initial side to avoid the oxidation of $\mathrm{Br}^{-}$by $\mathrm{ClO}_{2}$ during the soaking of the gel pieces. The kinetic role of $\mathrm{Br}^{-}$ ions is basically to consume $\mathrm{ClO}_{2}^{-}$ions according to reaction $\mathrm{R}^{2} .^{22}$

$$
\mathrm{HClO}_{2}+6 \mathrm{Br}^{-}+3 \mathrm{H}^{+} \rightarrow 2 \mathrm{Br}_{3}^{-}+\mathrm{Cl}^{-}+2 \mathrm{H}_{2} \mathrm{O}
$$

A considerable increase of wavelength has been observed in cases of both asymmetric initial $\mathrm{ClO}_{2}$ distributions, but in the case when $\mathrm{ClO}_{2}$ is added only to side $\mathrm{B}$, the patterns are less uniform and less visible (Figure 4a) than in the second case

(a)
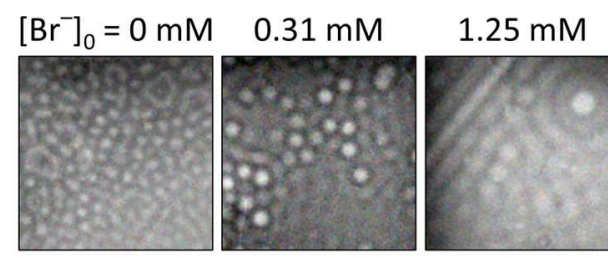

(b)
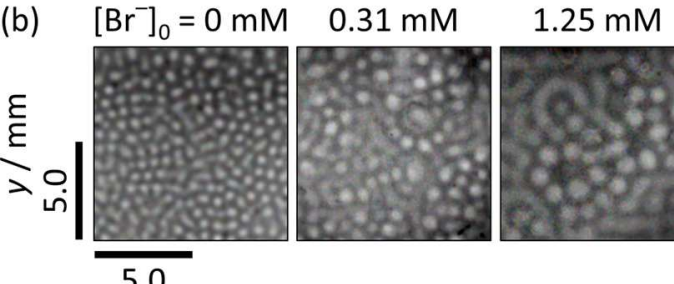

$$
x / \mathrm{mm}
$$
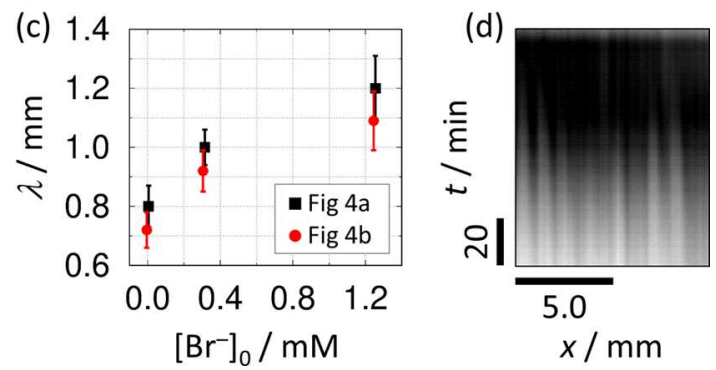

Figure 4. Results in the $\mathrm{TaB}$ experimental setup in the presence of $\left[\mathrm{Br}^{-}\right]_{0}$ which was added to the $\mathrm{ClO}_{2}$-free initial side. The evolving patterns are shown in the $x-y$ plane in the cases when $\mathrm{ClO}_{2}$ is added only to side $\mathrm{B}$ (a) and when $\mathrm{ClO}_{2}$ is added only to side A (b). The snapshots were made in different experiments at 55, 60, and $90 \mathrm{~min}$ (a) and at 60, 70, and 76 min (b), respectively. Dependence of the wavelength on the initial $\left[\mathrm{Br}^{-}\right]_{0}$ in the abovementioned cases (c). Temporal evolution of the stationary structures at $\left[\mathrm{Br}^{-}\right]_{0}=0.31 \mathrm{mM}$ when $\mathrm{ClO}_{2}$ is added only to side $\mathrm{B}(\mathrm{d})$. Concentrations: $[\mathrm{PVA}]_{0}=3$ g/L.

(Figure $4 \mathrm{~b}$ ). In the first case, the formation of stripes has been observed besides the spotlike pattern at $\left[\mathrm{Br}^{-}\right]_{0}=1.25 \mathrm{mM}$ (Figure 4a). The dependence of the resulting increased wavelength on the initial $\left[\mathrm{Br}^{-}\right]_{0}$ is almost the same in the case of both presented $\mathrm{ClO}_{2}$ distributions (Figure 4c). Figure $4 \mathrm{~d}$ shows the temporal evolution of the patterns in a selected case: the appearance of the stationary structures takes two times longer than in the bromide free case (Figure $3 \mathrm{~b}$ ), but the increased wavelengths are also constant in time for about 40 $\min$.

Besides the stationary Turing patterns, $\mathrm{RD}$ systems of the CDIMA reaction are also capable of producing time-periodic wave phenomena. According to the previous experimental observations, the formation of waves is expected at low PVA concentration. ${ }^{11}$ We have observed two different kinds of waves in the six experimental configurations at $[\mathrm{PVA}]_{0}=1 \mathrm{~g} /$ $\mathrm{L}$, without additional $\mathrm{Br}^{-}$ions. Type I waves are typical in the two asymmetric $\mathrm{ClO}_{2}$ distributions and appear at the beginning of the experiments as in-phase oscillations starting from several initial spots. On the time-space plot (Figure 5a),

(a)
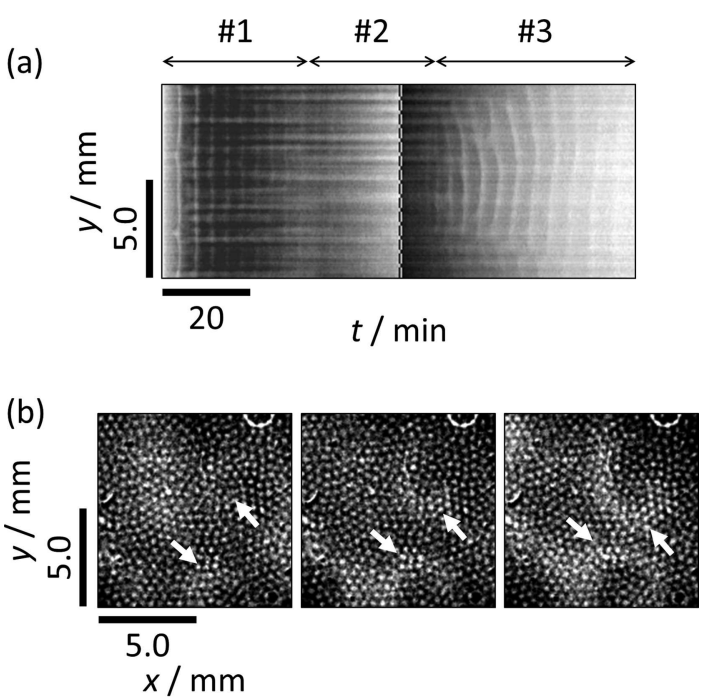

(c)

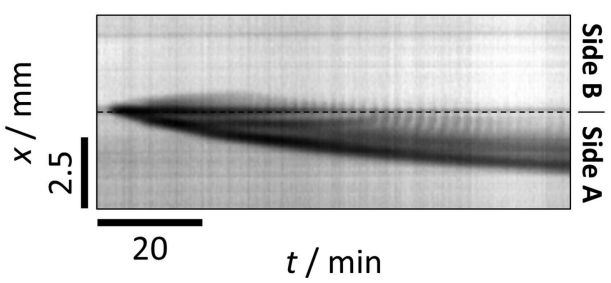

Figure 5. Waves at $[\mathrm{PVA}]_{0}=1 \mathrm{~g} / \mathrm{L}$ without adding $\mathrm{NaBr}$ to the initial solutions. Results in the $\mathrm{TaB}$ configuration: time-space plot in the case when $\mathrm{ClO}_{2}$ was added only to side $\mathrm{A}$ (a). The contrast was manipulated differently in the two sides separated by a dotted line. Sections \#1-3 indicate different types of behaviors. Interaction of spots and spiral waves on snapshots $\left(t_{1}=43 \mathrm{~min}, \Delta t=20 \mathrm{~s}\right)$ in the case when $\mathrm{ClO}_{2}$ was added to both sides (b). The waves are indicated by arrows. Results in the SbS configuration: layers of the oscillatory and Turing phenomena on a time-space plot made in the case when $\mathrm{ClO}_{2}$ was added to both sides (c).

these waves show up as parallel vertical lines with a period of $T$ $=3.8 \mathrm{~min}$ (beginning of section 1). During the first period of waves, the reaction zone is necessarily thin, and the Turing and Hopf instabilities occur in the same layer, therefore these modes strongly interact with each other.' Thus, the forming spots are erased by the wave at $t=4 \mathrm{~min}$, which is a full line in the time-space plot. As the reaction zone is getting wider, the interaction weakens and the waves and spots are formed separately along the direction of the concentration gradients. Accordingly, these structures are superimposed after $t=12$ min and oscillations appear as regularly spaced segmented lines in the time-space plot. In section 2, the oscillatory behavior vanishes, but the spotlike patterns remain stationary. In section 3 , the slow disappearance of Turing structures is accompanied by type II waves which are typical in all applied $\mathrm{ClO}_{2}$ distributions, both in $\mathrm{TaB}$ and $\mathrm{SbS}$ configurations. The period of them, which depends on the experimental setup, is almost the same as that of type I waves, but contrary to type I, these are spiral waves (Figure $5 b$ ), which annihilate when they 

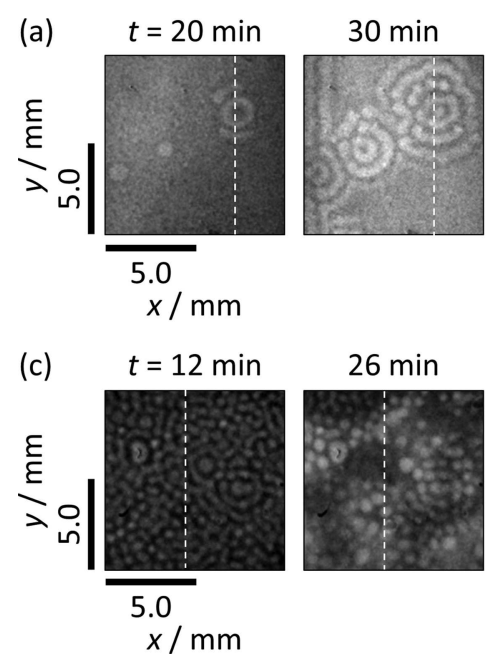

$50 \mathrm{~min}$

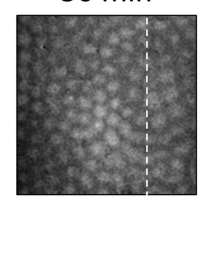

$58 \mathrm{~min}$

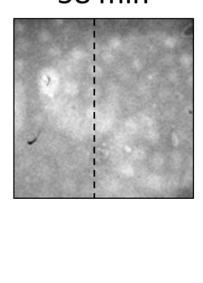

(b)

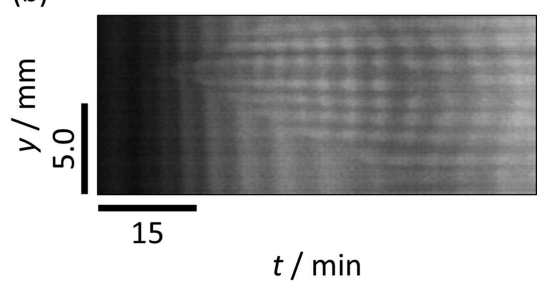

(d)

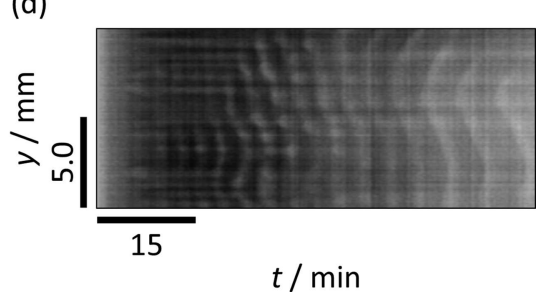

Figure 6. Results in the $\mathrm{TaB}$ geometry at $[\mathrm{PVA}]_{0}=1 \mathrm{~g} / \mathrm{L}$ in the presence $\left[\mathrm{Br}^{-}\right]_{0}$ which was added to the $\mathrm{ClO}_{2}$-free initial side. Snapshots $(\mathrm{a}, \mathrm{c})$ and time-space plots made along the dotted lines $(\mathrm{b}, \mathrm{d})$ in the case when $\mathrm{ClO}_{2}$ was added only to side $\mathrm{B}(\mathrm{a}, \mathrm{b})$ and in the case when $\mathrm{ClO}_{2}$ was added only to side A (c,d). Concentrations: $\left[\mathrm{Br}^{-}\right]_{0}=0.62 \mathrm{mM}(\mathrm{a}, \mathrm{b})$ and $\left[\mathrm{Br}^{-}\right]_{0}=0.31 \mathrm{mM}(\mathrm{c}, \mathrm{d})$.

collide. As it is shown in the $x-y$ representation of Figure $5 b$, the spirals may coexist with the Turing structures without obvious mutual perturbation. The interaction of these two phenomena has been investigated along the direction of the concentration gradients in the SbS geometry (Figure 5c). Comparing Figures $2 \mathrm{a}$ and $5 \mathrm{c}$, we can conclude that the waves and the last row of Turing spots are formed in different layers. In accordance with previous open gel reactor experiments, ${ }^{10}$ the waves appear toward side $\mathrm{B}$, where the local MA concentration values are higher. The unperturbed coexistence and the spatial separation of the oscillatory and stationary behaviors imply again a weak interaction between Turing and Hopf modes under the investigated conditions. ${ }^{9}$ By varying the initial concentration of $\mathrm{MA}$ in side $\mathrm{B}$, the stationary and oscillatory behaviors have been established separately. At small $[\mathrm{MA}]_{0}$ values only spots, at high $[\mathrm{MA}]_{0}$ only waves have been found.

We have investigated the combined effect of the smaller initial $[\mathrm{PVA}]_{0}$ and the addition of $\mathrm{Br}^{-}$ions to the $\mathrm{ClO}_{2}$-free side. The experiments were carried out in the $\mathrm{TaB}$ configuration in the cases of both asymmetric $\mathrm{ClO}_{2}$ distributions. As it has been expected from our previous results, we have seen waves and a remarkable increase of the wavelength of the stationary structures. However, as a new aspect, which was not reported before, we have found that the period of waves also increased during the experiments in the presence of $\mathrm{Br}^{-}$ions. In the case when $\mathrm{ClO}_{2}$ was added only to side $\mathrm{B}$, the stationary patterns have emerged from an oscillatory state through the formation of target-like patterns (Figure 6a). The wavelength of the resulting quasi-hexagonally ordered spots was $\lambda=0.97 \pm 0.09 \mathrm{~mm}$. In Figure $6 \mathrm{~b}$, at $t=20$ $\mathrm{min}$, it is clearly visible that the oscillations of the surrounding region do not penetrate to the domain of stationary patterns. At $t=30 \mathrm{~min}$, the waves become dominant in this region as well, which manifests as the superimposition of Turing and wave phenomena in the $x-y$ plane. The period of waves increased from $T=3.4$ to $5.4 \mathrm{~min}$ in $1 \mathrm{~h}$. However, in the case when $\mathrm{ClO}_{2}$ was added only to side $\mathrm{A}$, the wavelength of the Turing spots changed significantly during the measurement (Figure $6 \mathrm{c}, \lambda=0.68 \pm 0.07 \mathrm{~mm}$ at $t=12 \mathrm{~min}, \lambda=0.77 \pm 0.07$ $\mathrm{mm}$ at $t=26 \mathrm{~min}$ ). This alteration is connected to the intense wave phenomenon which started at $t=10 \mathrm{~min}$ in a localized domain (Figure 6d) with a period of $3.0 \mathrm{~min}$ and then spread in the whole setup, finally reaching the period of $5.3 \mathrm{~min}$. The existence of Turing patterns is shorter than in the previous case, and the disappearance of them is accompanied by the organization simple waves to spiral waves $(t=58 \mathrm{~min}$, Figure $6 c$ ). The special wave-spot behaviors (i.e., nonpenetrating waves and change in the wavelength) in the presence of smaller $[\mathrm{PVA}]_{0}$ and $\left[\mathrm{Br}^{-}\right]_{0}$ indicate a more expressed interaction between Turing and Hopf instabilities than in the previous cases.

\section{CONCLUSIONS}

We have created $\mathrm{RD}$ waves and Turing structures with the prototypical CDIMA reaction in an innovative gel reactor configuration. This is a closed two-layer gel geometry, which sets the initial condition on concentrations such that the active reactants are separated at $t=0$ between the two-halves of the system. This arrangement establishes antagonistic concentration gradients along the direction of diffusion, which is a universal route to pattern formation by means of the spatial localization of the undergoing reactions. ${ }^{23}$ The use of hydrogels as a medium allows us to study pure $\mathrm{RD}$ phenomena as the gel matrix avoids any macroscopic fluid motion. Accordingly, the effect of gravity plays no role in this reactor configuration. Naturally, in the above case only transient pattern formation can be observed. In the CDIMA reaction, because of the slow consumption of the initial reagents, this transient period is long enough to allow the development of different $\mathrm{RD}$ phenomena. Recently, these kinds of reactors have raised interest, and numerical works have predicted the existence of Turing and Hopf instabilities in them, when they can exhibit a transient batch oscillatory behavior. ${ }^{16,17}$ The predicted wave phenomena have been successfully reproduced in experiments with the $\mathrm{BZ}$ reaction, ${ }^{18}$ but here we show experimental verification for both the time-periodic and stationary behaviors. Our findings presented in time-space plots are in good agreement with previous simulated ones. ${ }^{16}$

A key point of this approach is the appropriate separation of the initial reactants to maintain well-defined initial and boundary conditions. In classic TSFR experiments, the reagents of the CIMA reaction are distributed as follows: 
$\mathrm{ClO}_{2}{ }^{-}$and $\mathrm{IO}_{3}{ }^{-}$are fed on one side while $\mathrm{MA}$ and $\mathrm{I}^{-}$are fed on the other. ${ }^{10}$ This arrangement results in gradually changing oxidizing capacity in the gel by the separation of the oxidant $\left(\mathrm{ClO}_{2}^{-}\right)$and the reductant (MA) and avoids any reactions at the boundaries. On the contrary, in our work we decided to use the CDIMA reaction as it is the minimal version of CIMA. In a closed system, it may be auspicious to choose the CDIMA variant to minimize the induction period. In this case, the analogue of the abovementioned distribution cannot be used since MA and $\mathrm{I}_{2}$ must be separated in different pools to avoid reaction $\mathrm{R} 1$. Our work confirmed that the applied initial distribution $\left(\mathrm{ClO}_{2}, \mathrm{I}_{2}, \mathrm{H}_{2} \mathrm{SO}_{4}, \mathrm{PVA} / / \mathrm{ClO}_{2}, \mathrm{MA}, \mathrm{H}_{2} \mathrm{SO}_{4}\right.$, and PVA) results in localized Turing patterns, parallel to the contact zone.

Our experimental findings verify the fact that the two-layer gel reactors are able to produce as diverse $\mathrm{RD}$ phenomena as have been generated in previous open reactor works in other reactor configurations. The two-layer setup creates crossgradients of reagents similar to the situation found in a twoside-fed open reactor. The closed configuration does not allow us to produce sustained patterns such as in a TSFR but because of the advantageous batch oscillatory properties of the CDIMA reaction, the transient batch dynamics develop for a period of time long enough to distinguish a large series of unusual stationary and dynamic patterns. In our experiments, the Turing patterns can be observed at least for $30 \mathrm{~min}$. This fact and the very significant simplicity of the experimental setup justify the application of the batch two-layer gel system. We confirm, in batch conditions, experimental evidence of the interaction of Turing and Hopf instabilities by using smaller initial amounts of PVA. ${ }^{9,26}$ The nature of this phenomenon depends on the width of the localized reaction zone, so it changes during one measurement. We have found that the waves appear in two distinct time domains which may be the result of the multiple and continuously changing concentration gradients. To clarify the origin of the arising instabilities, numerical simulations are needed. The dependence of the wavelength of the patterns on the addition of bromide ions was positively tested. We have observed a remarkable increase, not only in the case of stationary patterns but in the case of waves as well. This is in accordance with the previous result that the wavelength of Turing patterns is proportional to the square root of the periods of oscillations just beyond the Hopf bifurcation. $^{24}$

Because of its technical simplicity, the two-layer gel reactors can be appropriately used to establish demonstration experiments to illustrate the process of Turing-type pattern formation. The addition of bromide ions improves the visibility of the pattern by increasing its wavelength, which is also a desired feature in this context. It can be used not only with the CDIMA but with other nonlinear chemical reactions, for example, the $\mathrm{BZ}$ reaction ${ }^{18}$ and Landolt-type $\mathrm{pH}$ oscillators ${ }^{25}$ to study $\mathrm{RD}$ phenomena at special initial and boundary conditions. As an exclusive feature, it is easy to change the direction of observation in successive experiments because the switch between $\mathrm{SbS}$ and $\mathrm{TaB}$ geometries can be achieved without a hitch.

\section{EXPERIMENTAL METHODS}

Two pieces of gel, made of $2 \%$ agarose (Sigma-Aldrich, A2929) and loaded with different sets of chemicals of the CDIMA reaction, were joined to establish continuously changing cross-gradients of the reactants. The gels, with the dimensions of $16 \mathrm{~mm}\left(l_{x}\right) \times 30 \mathrm{~mm}\left(l_{y}\right) \times 2.8 \mathrm{~mm}\left(l_{z}\right)$, were put together in two different configurations (Figure 1e,f). The initial distribution of the chemicals was set by soaking the gels in stock solutions I and II for $3 \mathrm{~h}$ at $4{ }^{\circ} \mathrm{C}$ in the refrigerator. Solutions I and II were prepared by using primary $(\mathrm{a}-\mathrm{h})$ and secondary $(\mathrm{A}-\mathrm{D})$ solutions. Primary solutions: $(\mathrm{a})\left[\mathrm{H}_{2} \mathrm{SO}_{4}\right]=$ $0.04 \mathrm{M}$ [diluted from $1.0 \mathrm{~mol} / \mathrm{L}$ standard solution (VWR)], (b) $[\mathrm{PVA}]=12 \mathrm{~g} / \mathrm{L}$ (Aldrich, $80 \%$ hydrolyzed, $M_{\mathrm{w}}=9000-$ $10000),\left(\right.$ c) $\left[\mathrm{NaClO}_{2}\right]=0.016 \mathrm{M}$ (Fluka, $\left.\geq 80 \%\right),(\mathrm{d})$ $\left[\mathrm{K}_{2} \mathrm{~S}_{2} \mathrm{O}_{8}\right]=8 \times 10^{-3} \mathrm{M}$ (Reanal, alt.), (e) $\left[\mathrm{KIO}_{3}\right]=9.5 \times 10^{-4}$ $\mathrm{M}$ (Sigma-Aldrich, $\geq 98 \%$ ), (f) $[\mathrm{KI}]=4.56 \times 10^{-3} \mathrm{M}(\mathrm{VWR}$, $\geq 99 \%)$, (g) $[\mathrm{MA}]=0.02 \mathrm{M}$ (Fluka, $\geq 98 \%$ ), (h) deionized water. Secondary solutions were prepared by mixing unit volumes of primary solutions in the indicated order: (A) unit volumes of $(\mathrm{a}, \mathrm{c}, \mathrm{d})$ were mixed and then kept at $80{ }^{\circ} \mathrm{C}$ for 15 min, then the mixture was cooled to $4{ }^{\circ} \mathrm{C}$, then a unit volume of (b) was added, and finally this solution was kept at $4{ }^{\circ} \mathrm{C}$ in the refrigerator; (B) unit volumes of $(\mathrm{a}, \mathrm{b}, \mathrm{e}, \mathrm{f})$ were mixed; (C) unit volumes of $(a, b, g, h)$ were mixed; (D) unit volumes of $(\mathrm{a}, \mathrm{b})$ were mixed with double unit volume of $(\mathrm{h})$. Solutions $(B-D)$ were kept at room temperature after preparation. The secondary solutions were prepared freshly for every experiment. In the initial concentration distribution indicated in Figure 1a, solution I was prepared by mixing $4 \mathrm{~mL}(\mathrm{~A})$ and 16 $\mathrm{mL}$ (B), and solution II was prepared by mixing $4 \mathrm{~mL}$ (A), 6 $\mathrm{mL}(\mathrm{C})$, and $10 \mathrm{~mL}(\mathrm{D})$. In this case, both the stock solutions $\mathrm{I}$ and II contained equally $\left[\mathrm{H}_{2} \mathrm{SO}_{4}\right]=0.01 \mathrm{M},[\mathrm{PVA}]=3 \mathrm{~g} / \mathrm{L}$ and $\left[\mathrm{ClO}_{2}\right]=8 \times 10^{-3} \mathrm{M}$, which was produced in situ in the reaction of stoichiometric amounts of $\mathrm{K}_{2} \mathrm{~S}_{2} \mathrm{O}_{8}$ and $\mathrm{NaClO}_{2}$ (R6). Solution I also contained $\left[\mathrm{I}_{2}\right]=5.47 \times 10^{-4} \mathrm{M}$, which was produced in situ in the reaction of excess $\mathrm{KIO}_{3}$ and $\mathrm{KI}$ (R7), while solution II contained $[\mathrm{MA}]=1.5 \times 10^{-3} \mathrm{M}$.

$$
\begin{aligned}
& 2 \mathrm{ClO}_{2}^{-}+\mathrm{S}_{2} \mathrm{O}_{8}{ }^{2-} \rightarrow 2 \mathrm{ClO}_{2}+2 \mathrm{SO}_{4}{ }^{2-} \\
& \mathrm{IO}_{3}^{-}+5 \mathrm{I}^{-}+6 \mathrm{H}^{+} \rightarrow 3 \mathrm{I}_{2}+3 \mathrm{H}_{2} \mathrm{O}
\end{aligned}
$$

In the cases of Figure $1 \mathrm{~b}, \mathrm{c}$, solution (A) was replaced by (D) on the $\mathrm{ClO}_{2}$-free side. The addition of $\mathrm{Br}^{-}$to the $\mathrm{ClO}_{2}$-free side was implemented by replacing $(\mathrm{A})$ with $\left(\mathrm{D}^{\prime}\right)$, where $\left(\mathrm{D}^{\prime}\right)$ contained $\mathrm{NaBr}$ dissolved in (D). The concentrations of PVA and MA have been varied in the experiments, which was achieved by changing the concentrations of primary solutions $(\mathrm{b}, \mathrm{g})$ while the mixing ratios were the same in the following steps of the recipe. All solutions were prepared with ionexchanged water and chemicals were used without further purification. During the measurements, the gels were framed by a plastic cover to protect against desiccation. The measurements were carried out at $11^{\circ} \mathrm{C}$. The pictures were taken by an AVT Stingray F-033B (14 bit) camera through a $520 \mathrm{~nm}$ band pass filter to provide better contrast and recorded by the Streampix (Norpix) software. The camera was fixed above the experimental setup, which was enlightened from beneath by a $1 \mathrm{~mW} / \mathrm{cm}^{2}$ white light-emitting diode (LED) source. Higher irradiance may affect the pattern characteristics: ${ }^{12}$ using a $2 \mathrm{~mW} / \mathrm{cm}^{2}$ LED source, the lack of Turing patterns has been observed in our experiments. We used the ImageJ program for image processing.

\section{ASSOCIATED CONTENT}

\section{Supporting Information}

The Supporting Information is available free of charge on the ACS Publications website at DOI: 10.1021/acsomega.8b02997. 
Increase of the wavelength of these structures due to the addition of bromide ions (AVI)

Parallel formation of a hexagonal array of spots and spiral waves (AVI)

\section{AUTHOR INFORMATION}

\section{Corresponding Author}

*E-mail: szalai.istvan@chem.elte.hu (I.S.).

\section{ORCID}

Brigitta Dúzs: 0000-0002-0909-2808

István Szalai: 0000-0002-1859-1043

\section{Notes}

The authors declare no competing financial interest.

\section{ACKNOWLEDGMENTS}

The authors thank the support of the National Research, Development and Innovation Fund (119360) and the ÚNKP18-3 New National Excellence Program of the Ministry of Human Capacities.

\section{REFERENCES}

(1) van Roekel, H. W. H.; Rosier, B. J. H. M.; Meijer, L. H. H.; Hilbers, P. A. J.; Markvoort, A. J.; Huck, W. T. S.; de Greef, T. F. A. Programmable chemical reaction networks: emulating regulatory functions in living cells using a bottom-up approach. Chem. Soc. Rev. 2015, 44, 7465-7483.

(2) Zenk, J.; Scalise, D.; Wang, K.; Dorsey, P.; Fern, J.; Cruz, A.; Schulman, R. Stable DNA-based reaction-diffusion patterns. RSC Adv. 2017, 7, 18032-18040.

(3) Zadorin, A. S.; Rondelez, Y.; Gines, G.; Dilhas, V.; Urtel, G.; Zambrano, A.; Galas, J.-C.; Estevez-Torres, A. Synthesis and materialization of a reaction-diffusion French flag pattern. Nat. Chem. 2017, 9, 990.

(4) Lengyel, I.; Rabai, G.; Epstein, I. R. Experimental and modeling study of oscillations in the chlorine dioxide-iodine-malonic acid reaction. J. Am. Chem. Soc. 1990, 112, 9104-9110.

(5) De Kepper, P.; Dulos, E.; Boissonade, J.; De Wit, A.; Dewel, G.; Borckmans, P. Reaction-diffusion patterns in confined chemical systems. J. Stat. Phys. 2000, 101, 495-508.

(6) Castets, V.; Dulos, E.; Boissonade, J.; De Kepper, P. Experimental evidence of a sustained standing Turing-type nonequilibrium chemical pattern. Phys. Rev. Lett. 1990, 64, 2953.

(7) De Kepper, P.; Castets, V.; Dulos, E.; Boissonade, J. Turing-type chemical patterns in the chlorite-iodide-malonic acid reaction. Phys. $D$ 1991, 49, 161-169.

(8) Ouyang, Q.; Swinney, H. L. Transition from a uniform state to hexagonal and striped Turing patterns. Nature 1991, 352, 610-612.

(9) De Kepper, P.; Perraud, J.-J.; Rudovics, B.; Dulos, E. Experimental study of stationary Turing patterns and their interaction with traveling waves in a chemical system. Int. J. Bifurcation Chaos 1994, 04, 1215-1231.

(10) Rudovics, B.; Dulos, E.; de Kepper, P. Standard and nonstandard Turing patterns and waves in the ClMA reaction. Phys. Scr. 2006, T67, 43-50.

(11) Rudovics, B.; Barillot, E.; Davies, P. W.; Dulos, E.; Boissonade, J.; De Kepper, P. Experimental studies and quantitative modeling of Turing patterns in the (chlorine dioxide, iodine, malonic acid) reaction. J. Phys. Chem. A 1999, 103, 1790-1800.

(12) Horváth, A. K.; Dolnik, M.; Muñuzuri, A. P.; Zhabotinsky, A. M.; Epstein, I. R. Control of Turing structures by periodic illumination. Phys. Rev. Lett. 1999, 83, 2950-2952.

(13) Berenstein, I.; Yang, L.; Dolnik, M.; Zhabotinsky, A. M.; Epstein, I. R. Superlattice Turing structures in a photosensitive reaction-diffusion system. Phys. Rev. Lett. 2003, 91, 058302.
(14) De Kepper, P.; Epstein, I. R.; Kustin, K.; Orban, M. Batch oscillations and spatial wave patterns in chlorite oscillating systems. J. Phys. Chem. 1982, 86, 170-171.

(15) Lengyel, I.; Kadar, S.; Epstein, I. R. Transient Turing structures in a gradient-free closed system. Science 1993, 259, 493-495.

(16) Budroni, M. A.; De Wit, A. Localized stationary and traveling reaction-diffusion patterns in a two-layer $\mathrm{A}+\mathrm{B} \rightarrow$ oscillator system. Phys. Rev. E 2016, 93, 062207.

(17) Budroni, M. A.; De Wit, A. Dissipative structures: From reaction-diffusion to chemo-hydrodynamic patterns. Chaos 2017, 27, 104617.

(18) Budroni, M. A.; Lemaigre, L.; Escala, D. M.; Muñuzuri, A. P.; De Wit, A. Spatially localized chemical patterns around an A + B $\rightarrow$ oscillator front. J. Phys. Chem. A 2016, 120, 851-860.

(19) Lengyel, I.; Epstein, I. R. Modeling of Turing structures in the chlorite-iodide-malonic acid-starch reaction system. Science 1991, 251, $650-652$.

(20) Gaskins, D. K.; Pruc, E. E.; Epstein, I. R.; Dolnik, M. Multifold increases in Turing pattern wavelength in the chlorine dioxide-iodinemalonic acid reaction-diffusion system. Phys. Rev. Lett. 2016, 117, 056001.

(21) Dulos, E.; Davies, P.; Rudovics, B.; De Kepper, P. From quasi2D to 3D Turing patterns in ramped systems. Phys. D 1996, 98, 5366.

(22) Valdes-Aguilera, O.; Boyd, D. W.; Epstein, I. R.; Kustin, K. Systematic design of chemical oscillators. Part 35. Kinetics and mechanism of the reaction between chlorine(III) and bromide ion. J. Phys. Chem. 1986, 90, 6702-6708.

(23) Wolpert, L. Positional information and the spatial pattern of cellular differentiation. J. Theor. Biol. 1969, 25, 1-47.

(24) Ouyang, Q.; Li, R.; Li, G.; Swinney, H. L. Dependence of Turing pattern wavelength on diffusion rate. J. Chem. Phys. 1995, 102, 2551-2555.

(25) Dúzs, B.; Szalai, I. Front dynamics of $\mathrm{pH}$ oscillators with initially separated reactants. React. Kinet., Mech. Catal. 2017, 123, $335-349$.

(26) Boissonade, J.; Dulos, E.; De Kepper, P. Turing patterns: From myth to reality. In Chemical Waves and Patterns; Kapral, R., Showalter, K., Eds.; Springer: Dordrecht, 1995; Chapter 7, pp 221-268. 